\title{
Zoneamento hidroambiental da bacia hidrográfica do rio Acará, Amazônia Oriental
}

\author{
Hydro-environmental zoning of the hydrographic basin of Acará river, Eastern Amazon
}

\author{
Filipe Gomes Dias \\ Mestre em Ciências Ambientais pela Universidade \\ Federal do Pará (UFPA), Brasil \\ dias.filipe@live.com
}

Aline Maria Meiguins de Lima
Doutora em Desenvolvimento Sustentável do Trópico Úmido (UFPA)
Professora da Universidade Federal do Pará (UFPA), Brasil
alinemeiguins@gmail.com

\section{Resumo}

O zoneamento ambiental é um dos principais instrumentos de planejamento ambiental que contempla a análise integrada dos aspectos naturais e socioeconômicos, o qual permite identificar espacialmente as potencialidades e fragilidades dos sistemas ambientais. Nesse sentido, o presente trabalho teve como objetivo propor um zoneamento hidroambiental da bacia hidrográfica do rio Acará, no estado do Pará, sob um viés sistêmico e integrado da paisagem a partir do conhecimento das suas fragilidades, a fim de identificar as áreas que potencializam a manutenção de água no sistema hidrográfico, com intuito de preservação e conservação dos recursos hídricos. A metodologia baseouse na proposta de Ross para avaliar a fragilidade dos ambientes antropizados. Para isso, foram utilizados dados geocartográficos dos aspectos do meio físico e o padrão de uso e cobertura da terra com emprego da lógica Fuzzy para confeccionar as cartas de fragilidade hidropotencial e hidroambiental. Os resultados demonstram que o uso e cobertura da terra impactaram diretamente na manutenção de água no sistema hidrográfico da bacia, evidenciado através da conversão de classes com baixa fragilidade hidropotencial para classes com maior nível de fragilidade hidroambiental. Com o diagnóstico das condições hidroambientais da bacia do rio Acará, a proposta de zoneamento hidroambiental apresenta-se como um importante instrumento para os gestores no auxílio do processo de planejamento e reordenamento territorial.

Palavras-chave: paisagem, sistema hidrográfico, fragilidade hidropotencial e hidroambiental.

\begin{abstract}
Environmental zoning is one of the main instruments of environmental planning that contemplates the integrated analysis of natural and socioeconomic aspects, which allows identifying the potentialities and fragilities of environmental systems spatially. Thus, the present work aimed to propose hydro environmental zoning of the Acará river basin in the light of a systemic and integrated approach of the landscape based on the knowledge of its fragilities, in order to identify the areas that potentiate the maintenance of water in the hydrographic system, with the purpose of preservation and conservation of water resources. The methodology was based on Ross's proposal to evaluate the fragility of anthropised environments. For that, geocartographic data of the aspects of the physical environment and the land use and coverage pattern were used using Fuzzy logic to make hydropotential and hydro environmental fragility charts. With the diagnosis of the hydroenvironmental conditions of the Acará river basin, this proposal of hydro-environmental zoning is an essential instrument for the managers in the process of planning and territorial reorganisation. With
\end{abstract}


the diagnosis of the hydro-environmental conditions of the Acará river basin, this proposal of hydroenvironmental zoning is a vital instrument for managers, assisting in the process of planning and territorial reorganisation.

Keywords: landscape, hydrographic system, hydropotential and hydro-environmental fragility.

\section{INTRODUÇÃO}

O planejamento ambiental é de suma necessidade a fim de estabelecer bases para o (re)ordenamento territorial com o objetivo de corrigir e evitar problemas, e preservar e conservar os recursos naturais, por meio da compreensão de suas potencialidades a partir de uma perspectiva integrada da dinâmica natural e socioeconômica de determinado território. Nesse sentido, destaca-se o zoneamento ambiental, um dos principais instrumentos do planejamento ambiental (FONTES; PEJON, 2008; SANTOS; RANIERI, 2013).

O zoneamento ambiental consiste na ordenação do espaço em busca de sua otimização e das políticas públicas por meio da integração dos aspectos econômicos, sociais, ambientais, objetivando a utilização racional e sustentável dos recursos naturais (BECKER; EGLER, 1997). No entanto, Campos et al.. (2016) explica que apesar da importância desse instrumento no planejamento da paisagem, o mesmo não apresenta foco na dinâmica hídrica, e quando se propõe a tal objetivo a utilização da bacia hidrográfica como unidade de análise é fundamental.

A bacia reflete as intervenções antrópicas realizadas na dinâmica natural do meio físico e as possíveis alterações nos componentes do ciclo hidrológico, possibilitando, dessa forma, uma análise integrada dos aspectos socioeconômicos e naturais, como bem demanda as ações de planejamento ambiental.

O conceito de região hidrológica é relacionado a unidade de paisagem que tem a formação e transformação de seu modelado regulados pelo escoamento da água (BYCHKOV et al.., 2018). Trabalhos como os de Breña Naranjo et al.. (2011), Moulatlet et al.. (2015), Masud et al.. (2018), Rocha e Santos (2018) abordam os sistemas hídricos, as condicionantes do ciclo hidrológico e como as formas de uso e ocupação podem intervir nestas variáveis, reforçando assim a tese de que zonear sob a perspectiva da dinâmica hídrica na paisagem torna a bacia hidrográfica como unidade de análise essencial.

A metodologia proposta por Ross (1994) para avaliar a fragilidade ambiental dos ambientes modificados pelas ações antrópicas, fundamentada na concepção ecodinâmica de Tricart (1976), é uma abordagem que diagnóstica o grau de estabilidade (pedogênese) e instabilidade (morfogênese) da paisagem, o que orienta nas diretrizes do planejamento físico territorial, pois identificam e classificam unidades que apresentam grau elevado de homogeneidade. Nesse contexto, os estudos de 
diagnósticos e zoneamentos ambientais utilizam largamente tal proposta para análise e avaliação ambiental (FERREIRA, 2010).

Nesse sentido, o presente trabalho tem como objetivo propor um zoneamento hidroambiental da bacia hidrográfica do rio Acará, estado do Pará, sob um viés sistêmico e integrado da paisagem a partir do conhecimento das suas fragilidades, a fim de identificar áreas que potencializam a manutenção de água no sistema hidrográfico, com o intuito de preservação e conservação dos recursos hídricos.

\section{MATERIAIS E MÉTODOS}

\section{1. Área de Estudo}

A área de estudo do presente trabalho refere-se à bacia do rio Acará (Figura 1), no estado do Pará, que apresenta dimensão territorial de 13.537,24 $\mathrm{Km}^{2}$ e está localizada no Nordeste paraense. Abrangendo os municípios: do Acará, de Aurora do Pará, de Bujaru, de Concórdia do Pará, de Ipixuna do Pará, do Moju, de São Domingos do Capim, de Tailândia e de Tomé-Açu. Sendo que seu limite compreende cerca de 98\% da área dos municípios de Acará, Tailândia e Tomé-Açu.

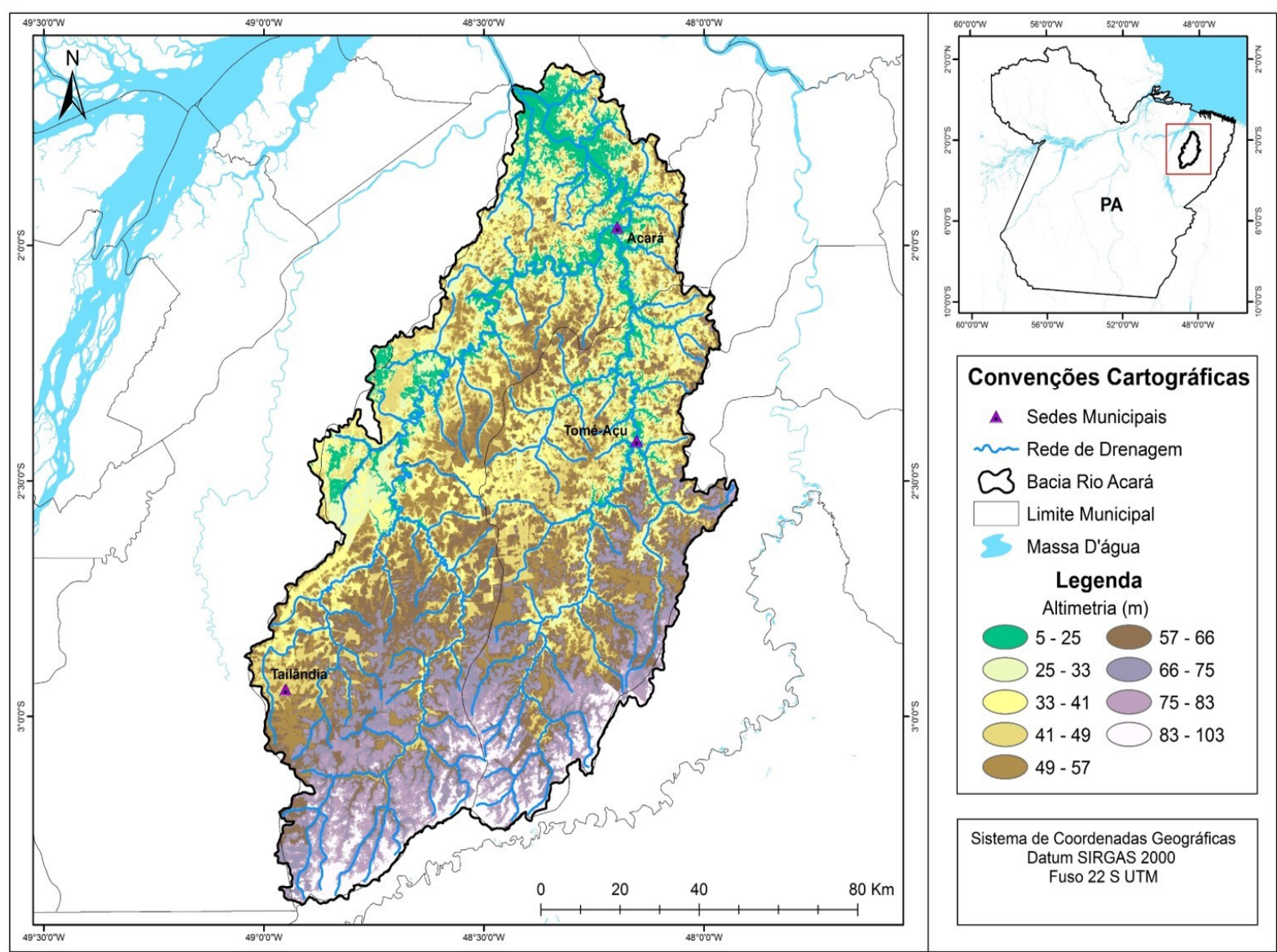

Figura 1 - Localização da bacia hidrográfica do rio Acará.

Fonte: Elaborado pelo autor, 2018. 
A fisiografia da bacia é caracterizada por substratos rochosos de material sedimentar formados no mesozoico e, principalmente, cenozoico. Geomorfologicamente, a bacia está inserida na unidade morfoestrutural do Planalto Rebaixados da Amazônia, formada por unidades morfológicas como planície fluvial, baixos platôs, tabuleiros e colinas, cujo relevo é plano e suavemente ondulado com cota altimétrica máxima de 103 metros. Os tipos de solos apresentam uma textura predominantemente argilosa, sendo o latossolo amarelo com maior distribuição na área da bacia (IBGE, 2003).

Os parâmetros morfométricos indicam que a bacia possui formato alongado, sendo a drenagem bem ramificada com padrão dendrítico de $4^{\mathrm{a}}$ ordem (DIAS; LIMA, 2018). Além disso, possui um elevado índice pluviométrico anual superior a $2.000 \mathrm{~mm}$ (ALBUQUERQUE et al.., 2010) que configura o potencial hídrico da região. A cobertura florestal da bacia é marcada pela presença de floresta ombrófila densa aluvial e de terra firme, além da vegetação secundária.

\subsection{Dados e Procedimentos metodológicos}

A utilização da proposta metodológica de Ross (1994) consiste na análise sistêmica dos componentes geoambientais da paisagem. Para isso, é necessário o compilamento e a produção de dados geoespaciais de tais componentes. A Figura 2 apresenta os componentes geoambientais instituídos na análise da fragilidade hidroambiental da bacia do rio Acará.

A obtenção dos dados geocartográficos digitalizados foi realizada em diversas instituições oficiais como: Instituto Brasileiro de Geografia e Estatística (IBGE), Instituto Nacional de Pesquisas Espaciais (INPE) e Agência Nacional das Águas (ANA). A escala dos dados temáticos estão em 1:100.000 e 1:250.000, onde a menor escala corresponde as informações regionais (geologia e pedologia).

O banco de dados geocartográficos foi sistematizado e processado no software de geoprocessamento ArcGis 10.1, no Laboratório de Estudos e Modelagem Hidroambientais (LEMHA) da Universidade Federal do Pará (UFPA), considerando o sistema de projeção oficial brasileiro SIRGAS 2000.

A confecção das cartas de geologia e tipos de solos foram realizadas a partir da base cartográfica do IBGE, escala 1:250.000, realizando a plotagem e, em seguida, o recorte das informações desses componentes de acordo com a delimitação da área da bacia do rio Acará.

O mapeamento geomorfológico da área da bacia do rio Acará foi realizado a partir da elaboração de cartas temáticas básicas - declividade, hipsométrica e curvas de nível, necessárias para a interpretação do relevo. Esses produtos foram gerados por meio de um Modelo Digital de Terreno 
(MDT). A partir disso, a taxonomia das formas de relevo seguiu a proposta estabelecida por Ross (1992).
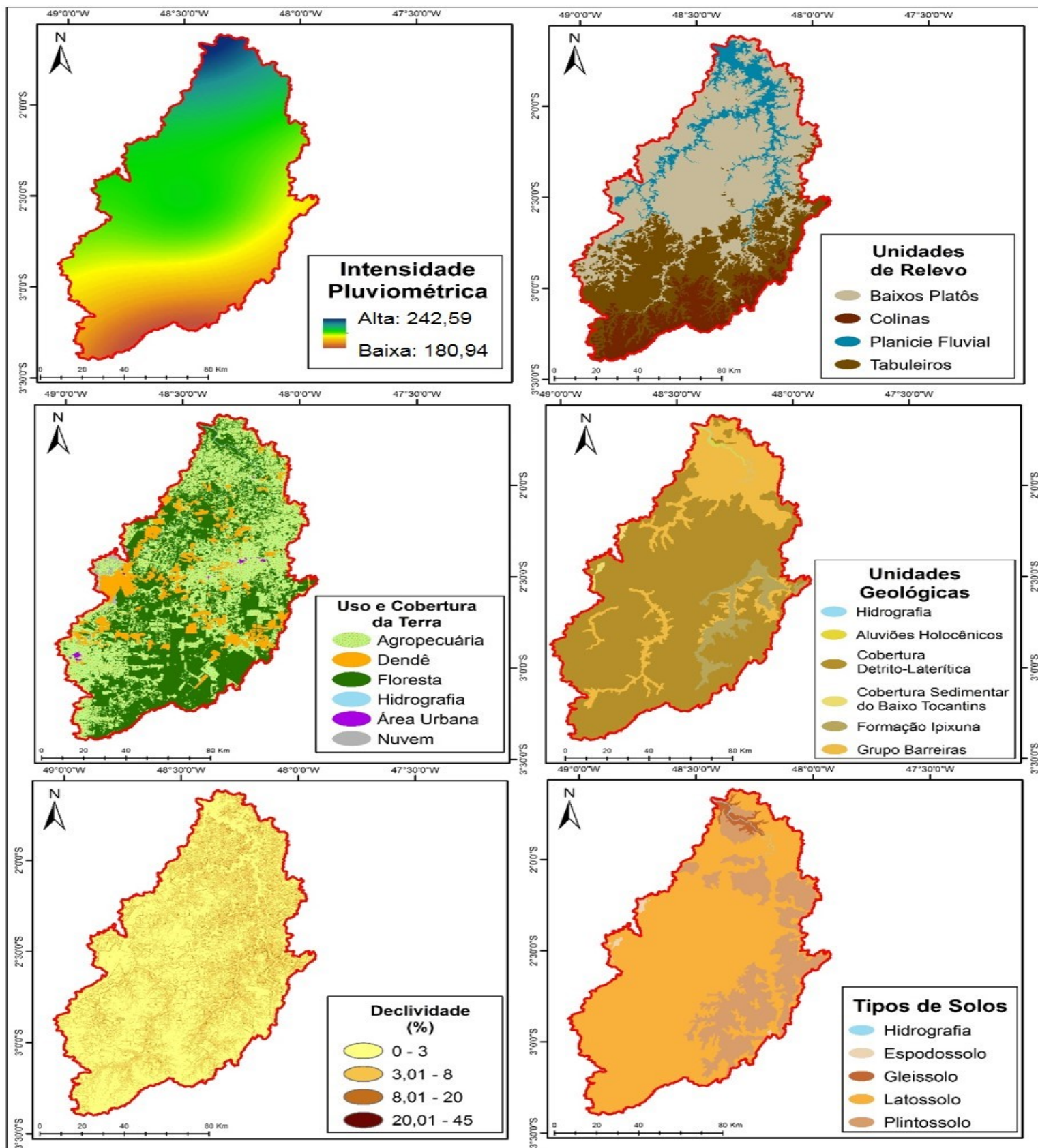

Figura 2 - Componentes geoambientais da bacia hidrográfica do rio Acará.

Fonte: Elaborado pelo autor, 2018.

A representação cartográfica da variável climática (intensidade pluviométrica) foi baseada em Bacani et al. (2015), onde os autores definem a intensidade pluviométrica como a relação entre a média da precipitação anual e o número de meses chuvosos durante o ano. O número de meses considerado para a região da bacia está de acordo com Crepani et al. (2001). As informações das 
médias anuais pluviométricas utilizadas dos últimos 30 anos (1988-2017) foram obtidas através do Global Precipitacion Climatology Centre (GPCC).

A espacialização da intensidade pluviométrica foi realizada a partir do método matemático de interpolação The Inverse Distance Weighted (IDW). De acordo com Bacani et al. (2015), o IDW é um interpolador determinístico univariado de médias ponderadas, a qual supõe que as feições adjacentes possuem características mais semelhantes do que as separadas.

O mapeamento do uso e cobertura da terra foi realizado a partir dos dados do Projeto de Monitoramento do Desmatamento da Floresta Amazônica Brasileira (PRODES) do INPE, do ano de 2017, onde foi reclassificada as classes do projeto (Desmatamento, Floresta, Hidrografia, Não Floresta, Nuvem, Resíduo) através da classificação visual, por meio de uma imagem Landsat 8 OLI, analisando suas características geométricas, tonalidade, cor e textura, para as classes propostas pelo presente trabalho. Após isso, foi realizada uma visita in loco no mês de junho de 2018 para subsidiar o processo de classificação visual.

A partir da instituição dos componentes geoambientais para análise da fragilidade hidroambiental, os atributos de tais componentes foram classificados de acordo com as características que potencializam a manutenção da água no sistema da bacia hidrográfica do rio Acará, ou seja, os atributos que potencializam a manutenção da água no sistema da bacia receberam peso próximo de 1 e os que não potencializam receberam peso de até 5 (Tabela 1). A atribuição dos pesos de fragilidade foi subsidiada pela literatura sobre o comportamento dos atributos dos componentes na entrada e saída da água no sistema da bacia hidrográfica, conforme exemplifica a Tabela 2.

Tabela 1 - Classes de fragilidade hidroambiental.

\begin{tabular}{cc}
\hline Classes de Fragilidade & Peso \\
\hline Muito Baixa & 1 \\
Baixa & 2 \\
Média & 3 \\
Alta & 4 \\
Muito Alta & 5 \\
\hline
\end{tabular}

Fonte: Elaborado pelo autor.

Após a classificação da fragilidade hidroambiental dos atributos foi realizada a padronização deles por meio da aplicação da lógica Fuzzy. Dentre a representação dos sistemas ambientais através da álgebra de mapas, essa é uma das principais aplicações utilizadas em diversos campos científicos, como a Geografia e as Ciências Ambientais. A lógica Fuzzy é definida como uma teoria matemática que trata de questões incertas, vagas, do raciocínio aproximado, que estão geralmente em linguagem natural e são convertidas para um formato numérico, o que possibilita o tratamento em ambiente computacional (KATINSKY, 1994; ZADEH, 1997). 
Tabela 2 - Literatura sobre o comportamento dos atributos dos componentes na entrada e saída da água no sistema da bacia hidrográfica.

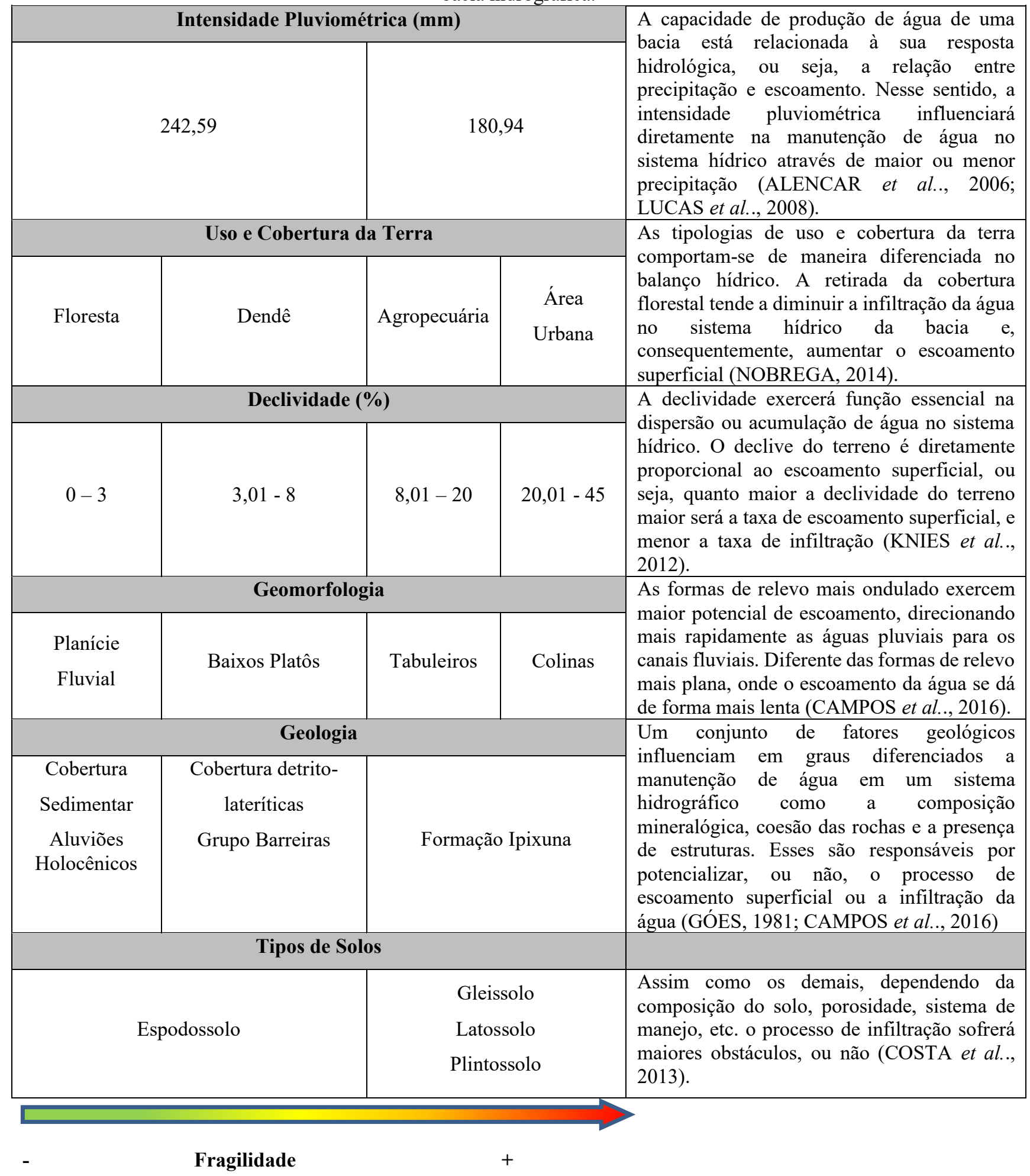

A lógica Fuzzy é caracterizada por incorporar as descontinuidades que marcam a real forma dos sistemas ambientais, mostrando-se bastante flexível. Nesse sentido, Burrough et al. (1992) explicam que a lógica Fuzzy é mais precisa que a lógica Booleana ${ }^{1}$ por ser mais flexível, ao invés de

\footnotetext{
1 “"...] envolve combinação lógica de mapas binários através de operadores condicionais. Cada mapa utilizado como uma condição pode ser entendido como um plano de informação (evidência). Os vários planos de informação são combinados
} 
aceitar intervalos rígidos, que acarreta a perda de informação e, consequentemente, resultados incompletos.

A aplicação da lógica Fuzzy pode ser utilizada metodologicamente para uma gama de trabalhos de cunho ambiental de diversos objetivos. Em função das vantagens proporcionadas por sua flexibilidade e incorporação das descontinuidades dos sistemas ambientais através de uma superfície numérica, diversos trabalhos como Silva (2005), Donha et al. (2006), De Paula et al. (2011), Cereda Junior e Röhm (2014), Bacani et al. (2015), demonstraram a eficiência da aplicação da lógica Fuzzy no mapeamento da fragilidade ambiental, apresentando resultados mais precisos na integração de diferentes critérios de análise. Nesse sentido, as ações adotadas para utilização do método Fuzzy estão descritas em Bacani et al. (2015).

Com o processo de fuzificação, os componentes geoambientais foram padronizados em uma superfície numérica que varia de 0 a 1 , sendo que os valores mais próximos de 0 favorecem a manutenção de água no sistema da bacia, e os valores próximos de 1 não sendo favoráveis.

Por último, antes da integração dos componentes geoambientais, foi realizada a ponderação dos componentes geoambientais através da técnica de tomada de decisão Analytic Hierarchy Process (AHP). De acordo com Câmara et al. (2001), a técnica AHP é uma teoria matemática voltada para a sistematização e avaliação da importância relativa entre diferentes critérios hierarquicamente, a qual busca medir as consistências dos julgamentos atribuídos. Nesse sentido, essa técnica consiste na atribuição de pesos, onde cada peso atribuído para as diferentes variáveis obedece ao potencial de determinada variável analisada (SAATY, 1977; SAATY, 1980).

A Figura 3 apresenta os componentes geoambientais fuzificados e os respectivos pesos AHP.

A Razão de Consistência, que avalia a técnica, do processo foi equivalente a 0,06 , o que significa que a AHP foi satisfatória, pois quanto mais próximo de 0 maior é a eficácia (SAATY, 1991).

Seguindo a abordagem de Ross (1994), foi elaborada a carta de fragilidade hidropotencial, ou seja, a sensibilidade dos componentes naturais (geologia, geomorfologia, tipos de solos, declividade e intensidade pluviométrica) em relação à manutenção de água no sistema da bacia do rio Acará. Portanto, foi realizada a integração desses diferentes componentes, com seus devidos pesos AHP, por meio de álgebra de mapas, conforme a Equação 1. A carta de fragilidade hidroambiental seguiu o mesmo procedimento, integrando com o uso e cobertura da terra, possibilitando, assim, analisar a intervenção das atividades antrópicas na dinâmica natural do sistema.

para dar suporte a uma hipótese ou preposição. O resultado é expresso de forma binária, “0” (hipótese não satisfeita) e "1" (hipótese satisfeita), não sendo possível a condição talvez." (CÂMARA et al, 2001, p. 9-8)" 


$$
F=\frac{\sum\left(n_{i} * p\right)}{n}
$$

Onde: $\mathrm{F}=$ fragilidade; $n_{i}=$ componentes naturais (para a Carta de Fragilidade Hidropotencial) ou componentes naturais e uso e cobertura da terra (para a Carta de Fragilidade Hidroambiental); $p=$ Peso AHP; $n=$ total de variáveis analisadas.

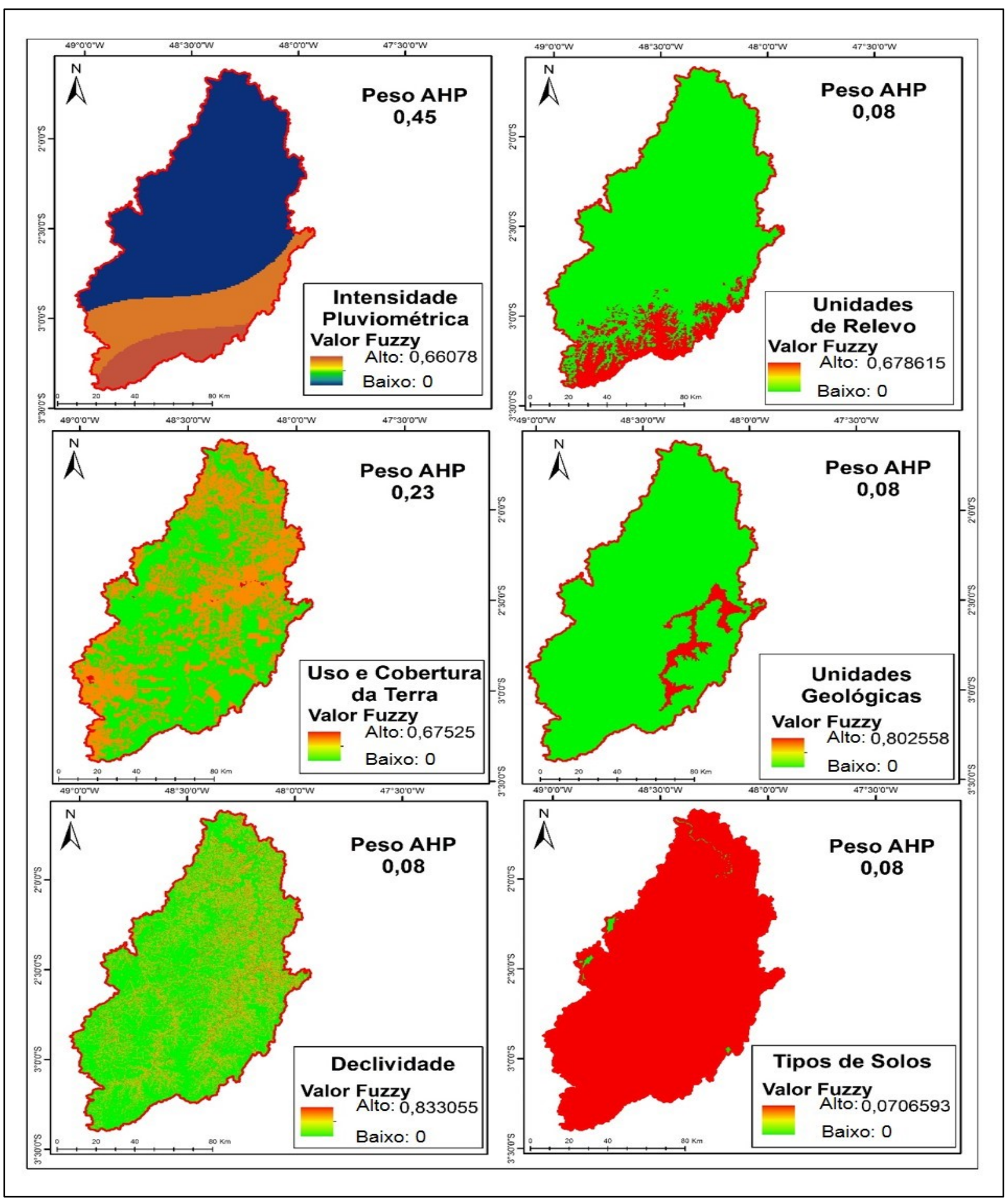

Figura 3 - Componentes fuzificados e pesos AHP.

Fonte: Elaborado pelo autor, 2018. 
O zoneamento hidroambiental foi elaborado através da integração entre a fragilidade hidroambiental e as zonas ripárias degradadas da bacia hidrográfica do rio Acará. A zona ripária da bacia do rio Acará foi obtida por meio do trabalho de Dias (2019), onde o autor detalha a metodologia de delimitação do ecossistema. A partir disso, foi analisada a intersecção entre a área da zona ripária e o uso da terra, o que possibilitou identificar as zonas degradadas. Por fim, foram classificadas quatro zonas em função da manutenção de água no sistema hidrográfico do rio Acará: Zona Favorável, Zona Média, Zona Desfavorável e Zona de Recuperação.

\section{RESULTADOS E DISCUSSÃO}

A carta de fragilidade hidropotencial foi produzida através da integração apenas dos componentes do meio físico (Figura 4), o que demonstra a sensibilidade inerente do sistema hidrográfico sem as intervenções das atividades antrópicas. Ela apresenta a classificação da fragilidade em muito baixa, baixa, média, alta e muito alta.

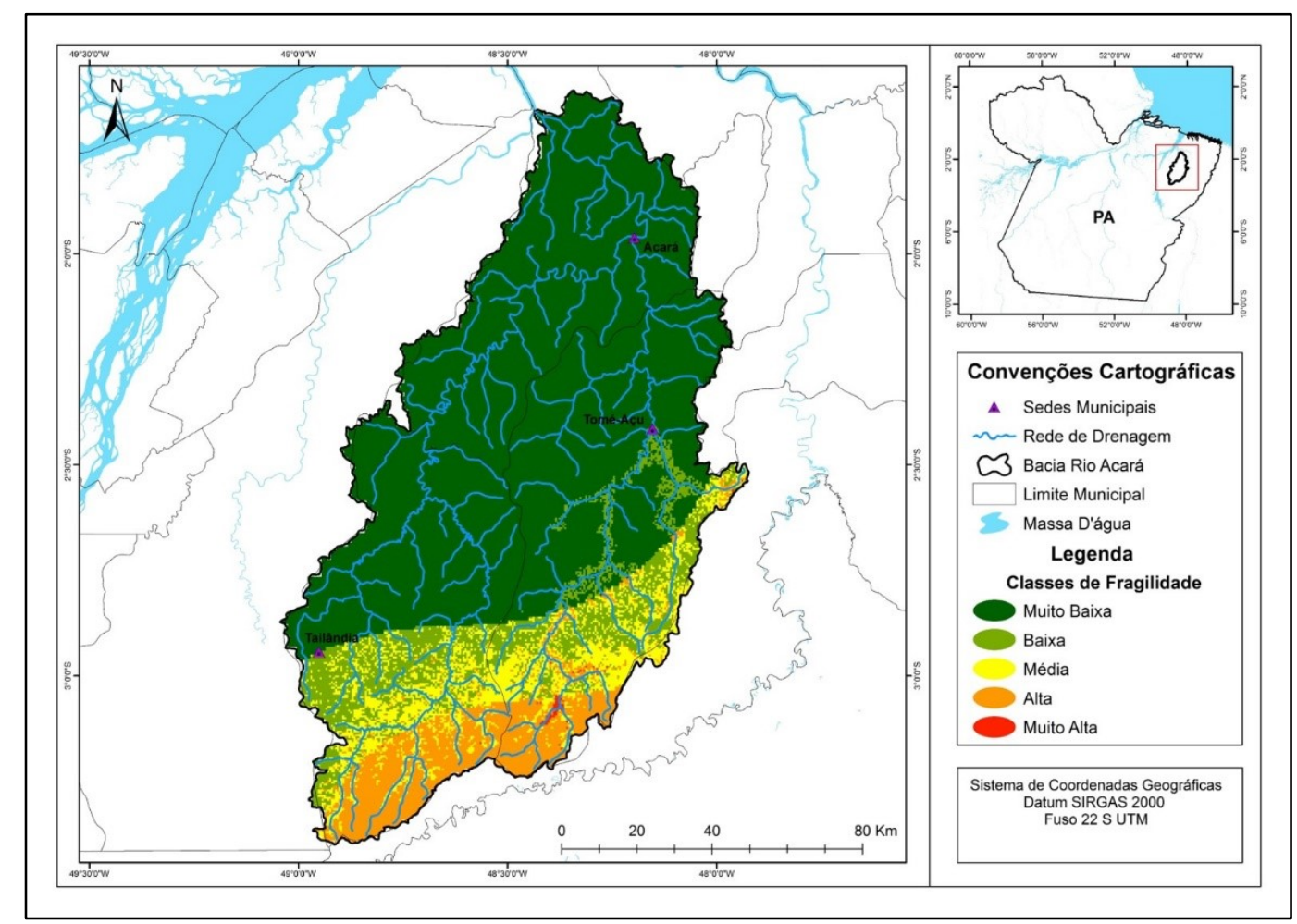

Figura 4 - Fragilidade hidropotencial da bacia do rio Acará.

Fonte: Elaborado pelo autor, 2018.

A carta de fragilidade hidroambiental foi confeccionada a partir da relação da fragilidade hidropotencial com o uso e cobertura da terra da bacia do rio Acará (Figura 5). Esse produto é de suma importância para ações de planejamento e gestão ambiental, pois permite identificar os níveis de intervenção que as ações antrópicas causam nos sistemas ambientais, e assim traçar estratégias de preservação e conservação dos recursos naturais. 


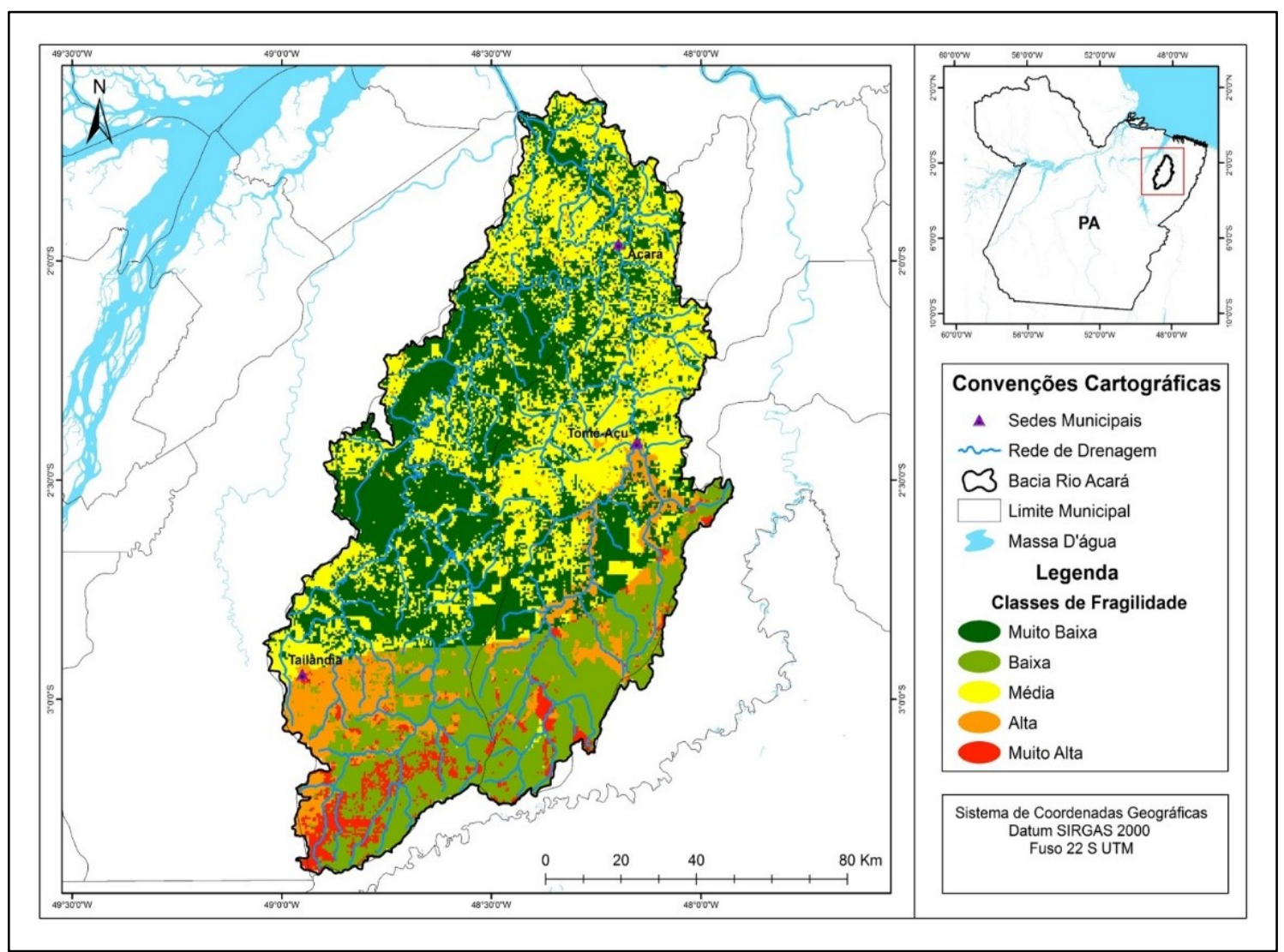

Figura 5 - Fragilidade hidroambiental da bacia do rio Acará. Fonte: Elaborado pelo autor, 2018.

A classe de fragilidade muito baixa predomina em ambas as cartas, no entanto, a carta de fragilidade hidroambiental apresenta uma diminuição substancial da mesma (Figura 6), evidenciando o grau de influência do processo de uso e ocupação do território da bacia. Sendo a tendência de crescimento do reflexo das alterações de uso e cobertura da terra observadas em todas as classes.

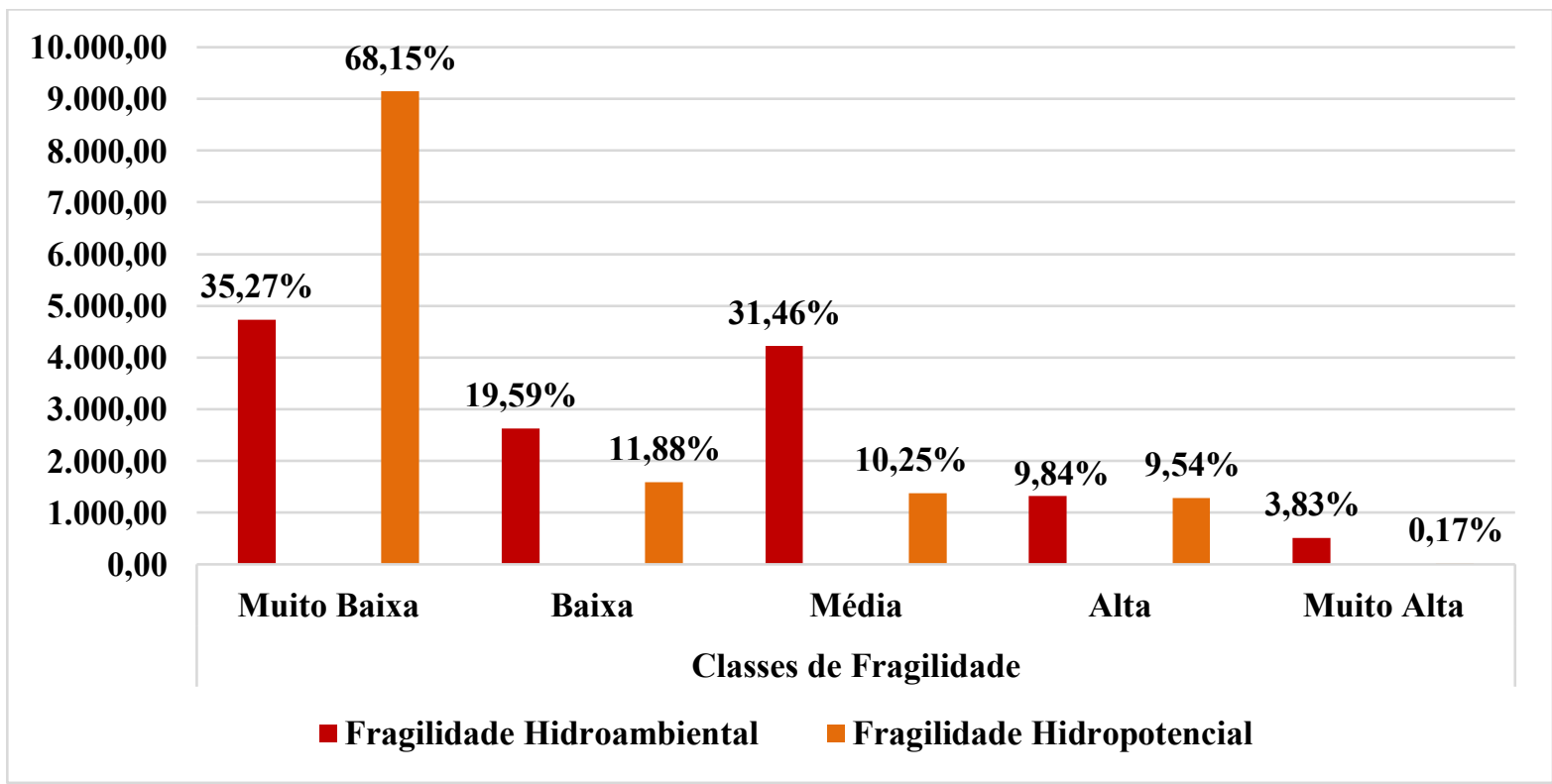

Figura 6 - Percentual das classes de fragilidade da bacia do rio Acará.

Fonte: Elaborado pelo autor. 
A predominância da classe muito baixa está relacionada ao conjunto dos componentes geoambientais que favorecem a manutenção da água no sistema hidrográfico do rio Acará, sobretudo a intensidade pluviométrica, que é bastante alta na região da área de estudo, ocorrendo maior probabilidade de recarga superficial e subterrânea.

As áreas de fragilidade hidropotencial muito baixa da bacia do rio Acará estão ligadas, além da intensidade pluviométrica maior, à formações geológicas de superfície e formas de relevo como cobertura detrito-laterítica e baixos platôs e tabuleiros, assim como um terreno majoritariamente plano, o que acaba por minimizar o escoamento superficial e favorecer o processo de infiltração da água.

A classe de fragilidade hidropotencial baixa corresponde a combinação dos mesmos componentes da classe anterior, entretanto a unidade geológica da formação Ipixuna, que apresenta uma sucessão de arenitos e siltitos, onde se caracteriza duas litofáceis. Uma inferior, composta por arenitos finos e siltitos, e outra superior formada por terrenos arenosos com intercalações de siltitos e horizontes de argila caulínicas (EMBRAPA, 2001), juntamente com um terreno moderadamente ondulado e intensidade pluviométrica menor, cuja infiltração no sistema hidrográfico sofrerá mais resistência em relação às áreas de classe muito baixa.

A fragilidade hidropotencial média possui a terceira maior distribuição espacial. Essa é definida diretamente pela diminuição da intensidade pluviométrica na região sudoeste e sudeste-leste da bacia, além das áreas com maiores altimetria formadas por formas de relevo do tipo colinas, assim como a presença dos latossolos, que são solos com textura argilosa. Portanto, a redução da intensidade pluviométrica, formas de relevo mais ondulado e solos com textura argilosa acaba minimizando o potencial de manutenção da água nessas áreas.

A menor intensidade pluviométrica de toda área da bacia irá ser prepoderante para as classes de fragilidade hidropotencial alta e muito alta. Dessa forma, a conjunção das formas de relevo ondulado, solos com textura argilosa, uma formação geológica de superfície com horizonte argiloso e menos chuva favorece o escoamento superficial, e faz com que a probabilidade de a água infiltrar no sistema hidrográfico do rio Acará seja substancialmente menor em relação às outras partes da bacia. Entretanto, essas áreas ocupam menos de 10\% do total.

Barker et al. (2016) destacam a importancia de se obsevar a influência do comportamento da precipitação pluviométrica na bacia hidrográfica considerando as propriedades de captação e propagação do escoamento. Gao et al. (2011) contextualizam a mesma influência já com a presença das formas de uso da terra que ampliam os efeitos já esperados da associação entre a paisagem natural e o efeito das chuvas, principalmente nas áreas de maior influência da rugosidade do relevo.

A menor fragilidade hidropotencial favorece tanto a recarga superficial quanto a subterrânea, como pode ser observado em Durães e Mello (2013). Owor et al. (2009) mantém o mesmo argumento, 
porém com ênfase nas águas subterrâneas, discutindo os efeitos da sazonalidade nestas. A análise diferenciando o período menos chuvoso do mais chuvoso é necessário principalmente nas bacias hidrográfias em que esta variação é bem marcada em termos de vazões, o que não pode ser verificado para a bacia do rio Acará, em função da ausência de dados fluviométricos. Porém, os trabalhos de: Gomes et al.. (2018), para a bacia do rio Capim; Ferreira et al. (2017) para a bacia do rio Moju; Santos et al. (2016) para a bacia do rio Xingu; e, Santos et al. (2014) para a bacia do rio Tapajós, apresentam esta influência para as bacias da região.

A análise da carta de fragilidade hidroambiental possibilita identificar a intensidade que o uso e cobertura da terra proporciona na manutenção da água no sistema hidrográfico do rio Acará. Esse efeito pode ser verificado na conversão das classes de fragilidade hidropotencial para classes de fragilidade hidroambiental, conforme aponta a Figura 7.

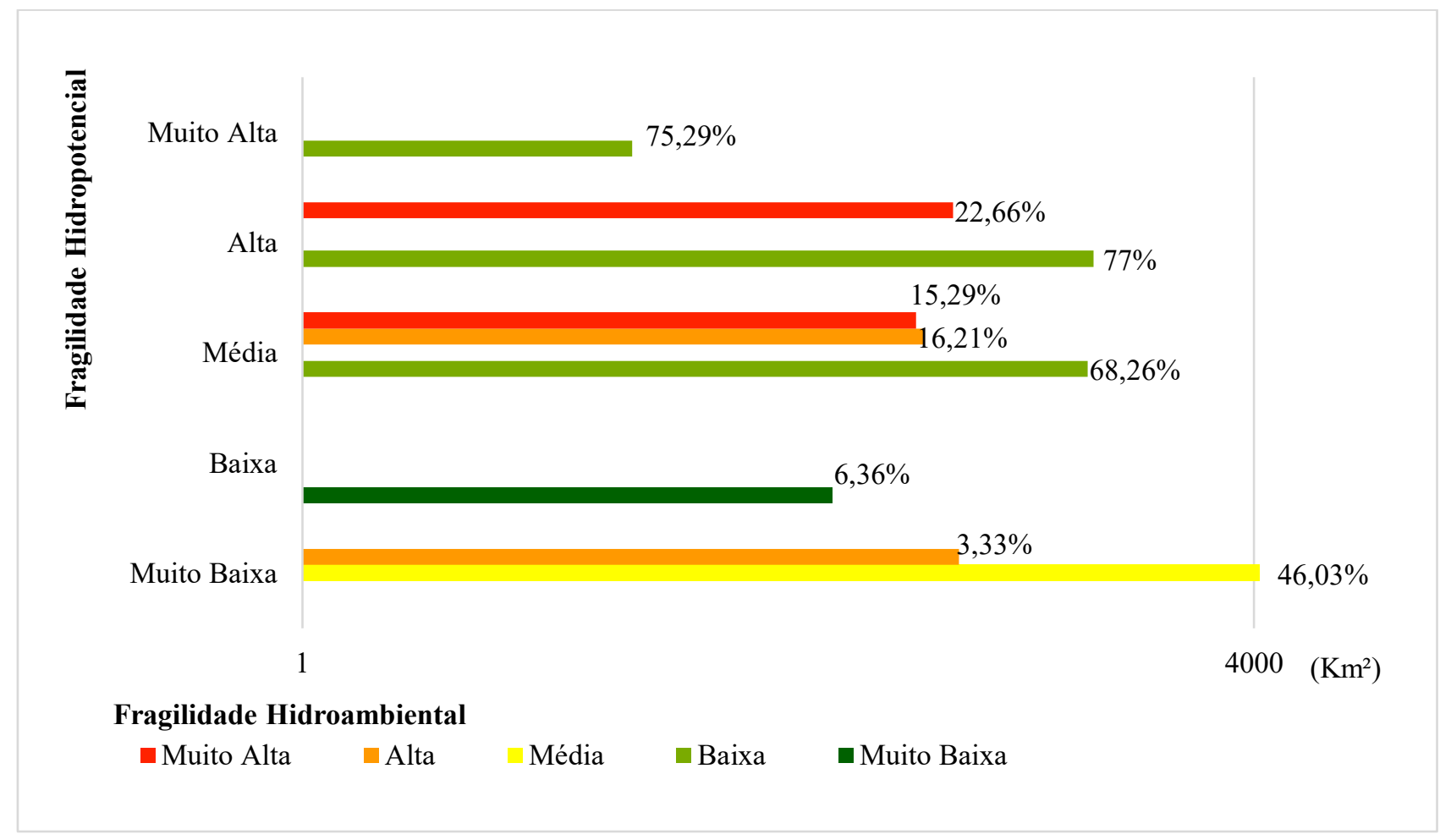

Figura 7 - Percentual da conversão das classes de fragilidade hidropotencial para hidroambiental da bacia do rio Acará.

Fonte: Elaborado pelo autor.

O uso e cobertura da terra alterou significativamente a dinâmica de manutenção da água no sistema hidrográfico do rio Acará. As áreas de classe muito baixa na carta de fragilidade hidropotencial passaram quase a metade para classes de fragilidade hidroambiental média (46,03\%) e alta (3,33\%). Isso decorre, principalmente, por meio das atividades agropecuárias como a pecuária bovina extensiva e, em menor grau, o núcleo urbano da sede municipal de Acará.

As áreas de fragilidade hidropotencial baixa sofreu poucas mudanças com a introdução do uso e cobertura da terra, fato decorrido pela presença da cobertura vegetal, que acaba por potencializar 
a infiltração no sistema hidrográfico do rio Acará com o aumento de áreas de fragilidade hidroambiental muito baixa $(6,36 \%)$.

Uma mudança significativa foi a conversão de áreas de fragilidade hidropotencial média para a classe de fragilidade hidroambiental baixa $(68,28 \%)$. A cobertura florestal é o principal fator da redução do nível de fragilidade, haja vista que, na mesma porção da bacia, as atividades agropecuárias, núcleos urbanos, relevo ondulado e solo com textura argilosa intensificaram os níveis de fragilidade hidroambiental alta $(16,21 \%)$ e muito alta $(15,29 \%)$.

A função da cobertura florestal na regulação dos fluxos d'água é importante de tal maneira, que a fragilidade hidropotencial alta foi convertida expressivamente em níveis de fragilidade hidroambiental baixa (77\%) após a correlação espacial com os outros componentes geoambientais. Por outro lado, a presença de atividades agropecuárias combinadas com áreas de relevo ondulado e menor intensidade pluviométrica a probabilidade de a água escorrer é maior. Relação essa que intensificou as áreas de fragilidade hidroambiental muito alta $(22,66 \%)$.

Assim como a conversão da fragilidade hidropotencial alta para fragilidade hidroambiental baixa, a transformação do nível de fragilidade hidropotencial muito alta para fragilidade hidroambiental baixa $(75,29 \%)$ se deu, primordialmente, pelo desempenho da cobertura florestal no processo de infiltração da água.

A partir da integração das informações de fragilidade hidroambiental com o uso e cobertura e os limites da zona ripária foi elaborada a carta de zoneamento hidroambiental da bacia hidrográfica do rio Acará. A carta apresenta quatro zonas hidroambientais, conforme demonstra a Figura 8.

A Zona Favorável está relacionada às áreas com fragilidade hidroambiental muito baixa e baixa. Ela é decorrente da cobertura florestal que está concentrada, principalmente, no alto curso da bacia. A presença da cobertura florestal nessa área é de suma importância, haja vista é uma área vital para a saúde hídrica da bacia, sobretudo, nas cabeceiras, pois é onde estão as nascentes dos principais rios, como o Acará e Acará-Mirim. Dessa forma, a probabilidade de a água infiltrar e favorecer a manutenção de água no sistema hídrico da bacia do rio Acará é maior nessa zona.

A Zona Média corresponde aos níveis de fragilidade hidroambiental média. Ela é caracterizada pela presença de atividades agropecuárias, principalmente áreas de pastos, que acabam por compactar o solo, com o pisoteio do gado; e, acabam por intensificar o processo de escoamento superficial e, consequentemente, contribui para a diminuição da infiltração de água no solo. Cabe destacar que o terreno é predominantemente plano e acaba por não contribuir para tal dinâmica, criando assim zonas intermediárias. 


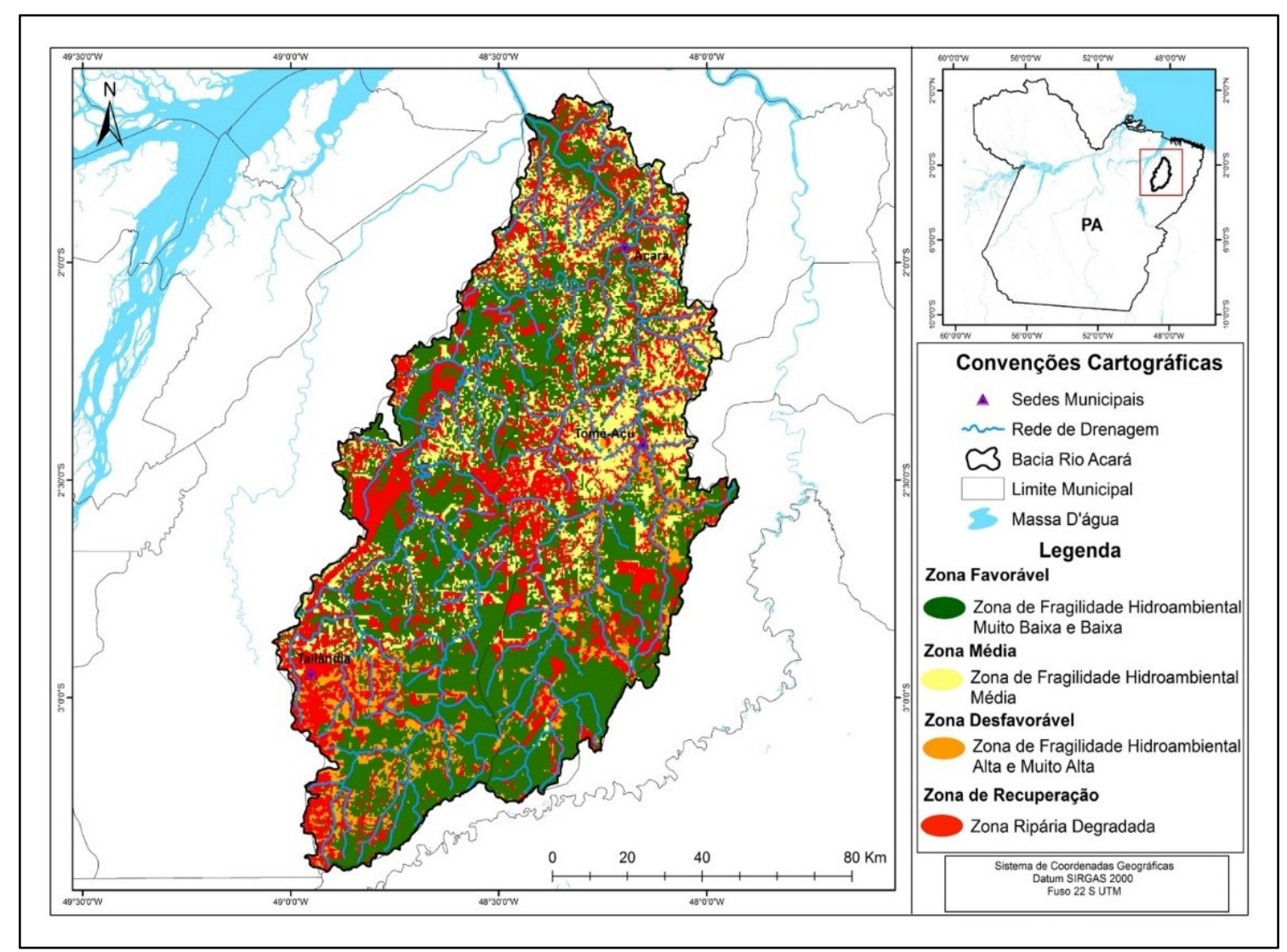

Figura 8 - Zoneamento hidroambiental da bacia do rio Acará.

Fonte: Elaborado pelo autor, 2018.

A Zona Desfavorável consiste nas áreas de fragilidade hidroambiental alta e muito alta. Caracteriza-se pela combinação de formação geológica e solo com textura argilosa intermediária. Áreas com declividade ondulada, atividades agropecuárias e menor intensidade pluviométrica, ou seja, áreas que apresentam naturalmente obstáculos, sendo ainda compactadas pelo pisoteio do gado e o menor índice pluviométrico, cujo processo de infiltração será menor em relação às outras zonas, haja vista que tal dinâmica favorece o escoamento superficial, e logo desfavorece a manutenção da água no sistema hidrográfico do rio Acará.

A Zona de Recuperação refere-se ao uso da terra nas zonas ripárias. Essas zonas são áreas hidrologicamente sensíveis que desempenham funções vitais na conservação dos recursos hídricos e da biodiversidade (ATTANASIO et al., 2012). A descaracterização dessas áreas deu-se por meio de atividades econômicas, sobretudo, aquelas sem práticas conservacionistas, pode comprometer seriamente a sustentabilidade hídrica da bacia hidrográfica. Portanto, faz-se necessário a inclusão desse ecossistema nas ações de planejamento e gestão ambiental para recuperar e conservar suas funções hidroecológicas. 


\section{CONSIDERAÇÕES FINAIS}

A análise da relação integrada dos componentes geoambientais da paisagem demonstrou que o uso e cobertura da terra influenciam diretamente na manutenção de água no sistema hidrográfico do rio Acará, pois considerando apenas a fragilidade hidropotencial os resultados apontam quase $80 \%$ da área total da bacia com as classes de fragilidade muito baixa e baixa.

Considerando a intervenção antrópica, por meio das atividades agroeconômicas, o quadro hidroambiental não apresenta, nessa perspectiva, condições críticas, pois ainda prevalece um pouco mais de $50 \%$ da área da bacia com as classes de fragilidade muito baixa e baixa. Entretanto, o aumento substancial da classe de fragilidade média alerta para a necessidade de controle e monitoramento da expansão de tais atividades, principalmente para áreas que desempenham funções hidroecológicas primordiais, como as zonas ripárias ao longo dos cursos e cabeceiras dos rios. Além disso, faz-se necessário a recuperação das áreas que já passaram pelo processo de degradação.

Com o diagnóstico das condições hidroambientais da bacia do rio Acará, a proposta de zoneamento hidroambiental apresenta-se como um importante instrumento para os gestores no auxílio do processo de planejamento e (re)ordenamento territorial e, consequentemente, a tomada de decisão, cujo objetivo deve ser a recuperação dos recursos naturais degradados e a preservação e conservação daqueles não alterados diretamente.

A proposta metodológica integrada utilizada de avaliação da fragilidade hidroambiental demonstrou-se satisfatória, a qual possibilitou identificar o grau de intervenção que a ação antrópica impõe aos sistemas ambientais. Tal metodologia é potencializada com o emprego das ferramentas de geotecnologias, que propicia o processamento de um conjunto de dados com rapidez e precisão, resultando em importantes informações geoespaciais. Ressalta-se, também, a importância da adoção do método Fuzzy nessa abordagem, pois ele ultrapassa a rigidez representativa dos sistemas ambientais apresentado pela abordagem booleana, demonstrando maior flexibilidade e resultados mais completos.

A metodologia utilizada possui alto potencial de análise e avaliação ambiental e pode ser replicada para outras áreas. No que se refere a esta pesquisa, o presente trabalho apresenta algumas lacunas, por exemplo, a inserção de dados de evapotranspiração e de vazão para a área de estudo, com isso poderiam ser obtidos resultados mais exatos e sólidos; porém com a potencialidade para desbramentos e adicionamentos em pesquisas e resultados futuros. 


\section{REFERÊNCIAS}

ALBUQUERQUE, M. F.; SOUZA, E. B.; OLIVEIRA, M. C. F.; SOUZA JÚNIOR, J. A. Precipitação nas mesorregiões do estado do Pará: climatologia, variabilidade e tendências nas últimas décadas (1978-2008). Revista Brasileira de Climatologia, Curitiba, v. 6, p. 151-168, 2010.

ALENCAR, D. B. S.; SILVA, C. L.; OLIVEIRA, C. A. Influência da precipitação no escoamento superficial em uma microbacia hidrográfica do Distrito Federal. Eng. Agrícola, Jaboticabal, v. 26, n. 1, p. 103-112, 2006.

ATTANASIO, C. M.; GANDOFI, S.; ZAKIA, M. J. B.; VENIZIANI JUNIOR, J. C. T.; LIMA, W. P. A importância das áreas ripárias para a sustentabilidade hidrológica do uso da terra em microbacias hidrográficas. Bragantina, Campinas, v. 71, n. 4, p. 493-501, 2012.

BACANI, V. M.; SAKAMOTO, A. Y.; LUCHIARI, A.; QUÉNOL, H. Sensoriamento remoto e SIG aplicados à avaliação da fragilidade ambiental de bacia hidrográfica. Mercator, Fortaleza, v. 14, n. 2, p. 119-135, 2015.

BARKER, L. J.; HANNAFORD, J.; CHIVERTON, A.; SVENSSON, C. From meteorological to hydrological drought using standardised indicators. Hydrology and Earth System Sciences Discussions, v. 12, n. 1, p. 2827-2875, 2015.

BECKER, B. K.; EGLER, C. Detalhamento da Metodologia para Execução do Zoneamento Ecológico Econômico pelos estados da Amazônia. Rio de Janeiro: SAE - Secretaria de Assuntos Estratégicos/Ministério do meio Ambiente. 1997.43p.

BREÑA NARANJO, J. A.; WEILER, M.; STAHL, K. Sensitivity of a data-driven soil water balance model to estimate summer evapotranspiration along a forest chronosequence. Hydrol. Earth Syst. Sci., v. 15, p. 3461-3473, 2011.

BURROUGH, P. A.; MACMILLAN, P. A.; VAN DEURSEN, W. Fuzzy classification methods for determining land suitability from soil profile observation and topography. Journal of Soil Science, v. 43, n. 2, p. 193-210, 1992.

BYCHKOV, I. V.; GAGARINOVA, O. V.; ORLOVA, I. I.; BOGDANOV, V. N. Water Protection Zoning as an Instrument of Preservation for Lake Baikal. Water, v. 10, p. 1-12, 2018.

CÂMARA, G.; DAVIS, C. Introdução à Ciência da Geoinformação. $2^{\mathrm{a}}$ Edição. São José dos Campos: INPE, 2001 disponível em: http://www.dpi.inpe.br/gilberto/livro/. Acesso em: 15 jun. 2018.

CAMPOS, D. O.; SANTOS, J. W. B.; SILVA, E. V.; REGO, N. A. C. Zoneamento geohidroecológico da bacia do rio Almada: Análise da capacidade de produção de água. In: MORAES, M. E. B.; LORANDI, R. Métodos e técnicas de pesquisa em bacias hidrográficas. Ilhéus: Editus, 2016. p. 81-101.

CEREDA JUNIOR, A.; RÖHM, S. A. Analysis of environmental fragility using multi-criteria analysis (MCE) for integrated landscape assessment. Journal of Urban and Environmental Engineering, v. 8, n. 1, p. 28-37, 2014.

COSTA, C. F. G.; FIGUEIREDO, R. O.; OLIVEIRA, F. A.; SANTOS, I. P. O. Escoamento Superficial em Latossolo Amarelo Distrófico Típico sob diferentes Agrossistemas no Nordeste 
Paraense. Revista Brasileira de Engenharia Agrícola e Ambiental, Campina Grande, v. 17, n. 2, p. 162-169, 2013.

CREPANI, E.; MEDEIROS, J. S.; FILHO, P. H.; FLORENZANO, T. G.; DUARTE, V.; BARBOSA, C. C. F. Sensoriamento Remoto e Geoprocessamento Aplicados ao Zoneamento EcológicoEconômico e ao Ordenamento territorial. SAE/INPE, São José dos Campos, 2001. 103p.

DE PAULA, E. M.; SOUZA, M. J. N. de. Sistemas de Informações Geográficas na Análise da Vulnerabilidade Ambiental da Bacia do Rio Ceará-CE. Revista Brasileira de Cartografia, Brasília, v. 63 , n. 4, p. 515-525, 2011.

DIAS, F. G. Análise integrada da paisagem na bacia hidrográfica do rio Acará: subsídios ao planejamento ambiental. 2019. 132 f. Dissertação (Mestrado em Ciências Ambientais) - Instituto de Geociências, Universidade Federal do Pará, Belém, 2019.

DONHA, A. G; SOUZA, L. C. P; SUGAMOTO, M. L. Determinação da fragilidade ambiental utilizando técnicas de suporte à decisão e SIG. Revista Brasileira de Engenharia Agrícola e Ambiental, Campina Grande, v. 10, n. 1, p. 175-181, 2006.

DURÃES, M. F.; MELLO, C. R. Groundwater recharge behavior based on surface runoff hydrographs in two basins of the Minas Gerais state. Revista Ambiente \& Água - An Interdisciplinary Journal of Applied Science, v. 8, n. 2, p. 57-66, 2013.

EMBRAPA. Caracterização e classificação dos solos do município de Tomé-Açu, PA. Belém: Embrapa Amazônia Oriental, 2001. Disponível em: infoteca.cnptia.embrapa.br/handle/doc/403598. Acesso em: 15 jun. 2018.

FERREIRA, S. C. G.; LIMA, A. M. M. E; CORRÊA, J. A. M. Zoneamento da bacia hidrográfica do rio Moju (Pará): usos da água e sua relação com as formas de uso e cobertura do solo. Revista Ambiente \& Água - An Interdisciplinary Journal of Applied Science, Taubaté, v. 12, p. 680-693, 2017.

FERREIRA, V. O. A Abordagem da Paisagem no Âmbito dos Estudos Ambientais Integrados. GeoTextos, Salvador, v. 6, n. 2, p. 187-208, 2010.

FONTES, S. B.; PEJON, O. J. Proposal of a geo-environmental zoning method based on Ottobasin compartmentalisation. Bulletin of Engineering Geology and the Environment, v. 67, p. 555-563, 2008.

GAO, P.; MU, X.-M.; WANG, F.; LI, R. Changes in streamflow and sediment discharge and the response to human activities in the middle reaches of the Yellow River. Hydrology and Earth System Sciences, v. 15, n. 1, p. 1-10, 2011.

GÓES, A. M. Estudo Sedimentológico dos Sedimentos Barreiras, Ipixuna e Itapecuru no Nordeste do Pará e Noroeste do Maranhão. 1981. 55 f. Dissertação (Mestrado em Ciências Geofísicas e Geológicas) - Instituto de Geociências, Universidade Federal do Pará, Belém, 1981.

GOMES, D. J. C.; LIMA, M. M. A.; SILVA JUNIOR, J. A.; SERRÃO, E. A. O. Impactos das mudanças de uso da terra e eventos climáticos extremos em sistemas hidrológicos da Amazônia oriental - bacia do rio Capim (PA-MA). Revista Geográfica Acadêmica, Boa Vista, v. 12, p. 153$172,2018$. 
KATINSKY, M. Fuzzy set modelling in Geographical Information Systems. MsC Thesis, University of Wisconsin-Madsin, USA, 1994.

KNIES, A. E. et al.. Escoamento Superficial em diferentes Declividades do Terreno e Níveis de Cobertura da Superfície do Solo. In: WORKSHOP INTERNACIONAL DE ONOVAÇÕES TECNOLÓGICAS NA IRRIGAÇÃO. 4., 2012. Fortaleza. Anais... Fortaleza, 2012. p. 1-3.

LUCAS, E. W. M.; SOUZA, F. A. S.; F. D. S.; RAMOS, A. M.; CONDE, F. C. Influência do fenômeno ENOS na resposta hidrológica anual da sub-bacia Amazônica/Brasil (Região Hidrográfica do Xingu/Pará). Repositório AEMET, 2008.

MASUD, M. B.; FERDOUS, J.; FARAMARZI, M. Projected Changes in Hydrological Variables in the Agricultural Region of Alberta, Canada. Water, v. 10, p. 1-18, 2018.

MOUlATlet, G. M.; RENNÓ, C. D.; COSTA, F. R. C.; EMILIO, T.; SCHIETTI, J. Mapping hydrological environments in central Amazonia: ground validation and surface model based on SRTM DEM data corrected for deforestation. Earth Syst. Sci. Data, v. 7, p. 29-34, 2015.

NOBREGA, R. S. Impactos do desmatamento e de mudanças climáticas nos recursos hídricos na Amazônia ocidental utilizando o modelo SLURP. Revista Brasileira de Meteorologia, São José dos Campos, v. 29, p. 111-120, 2014.

OWOR, M.; TAYLOR, R.G.; TINDIMUGAYA, C.; MWESIGWA, D.; BOVOLO, C.I.; PARKIN, G.; SOPHOCLEOUS, M. Rainfall intensity and groundwater recharge: Empirical evidence from the Upper Nile Basin. Environmental Research Letters, v. 4, p. 1-6, 2009.

ROCHA, P. C.; SANTOS, A. A. Hydrological analysis in water basins. Mercator, Fortaleza, v. 17, p. $1-18,2018$.

ROSS, J. L. S. Análise empírica da fragilidade dos ambientes naturais e antropizados. Revista do Departamento de Geografia, São Paulo, v. 8, p. 3-74, 1994.

ROSS, J. L. S. O Registro cartográfico dos fatos geomorfológicos e a questão da taxonomia do relevo. Revista do Departamento de Geografia, São Paulo, v. 6, p. 17-29, 1992.

SAATY, T. L. A Scaling Method for Priorities in Hierarchical Structures. Journal of Mathematical Psychology, v. 15, p. 234-281, 1977.

SAATY, T. L. The Analytic Hierarchy Process: Planning, priority setting, resource allocation. 1. ed. New York: McGraw-Hill, 1980. 287p.

SAATY, T. L. Some Mathematical Concepts of the Analytic Hierarchy Process. Behaviormetrika, v. 29, p. 1-9, 1991.

SANTOS, C. A.; LIMA, A. M. M.; SERRÃO, E. A. O; FARIAS, M. H. C. S.; WANZELER, R. T. S.; ARAÚJO, I. B. Modelo de previsão fluviométrica para o rio Xingu na cidade de Altamira - PA. Revista Internacional de Ciências, Rio de Janeiro, v. 6, p. 230-243, 2016.

SANTOS, C. A.; SERRÃO, E. A. O.; GONCALVES, L. J. M.; WANZELER, R. T. S.; LiMA, A. M. M. Zoneamento da distribuição da precipitação pluviométrica na bacia hidrográfica do rio Tapajós. Enciclopédia Biosfera, v. 10, p. 3092-3106, 2014. 
SANTOS, M. R. R.; RANIEIRI, V. E. L. Critérios para análise do zoneamento ambiental como instrumento de planejamento e ordenamento territorial. Ambiente e Sociedade, Campinas, v. 16, p. 43-62, 2013.

SILVA, S. F. Zoneamento Geoambiental com Auxílio de Lógica Fuzzy e proposta de um Geoindicador para Caracterização do Meio Físico da bacia do Rio do Peixe. 2005. 394 f. Tese (Doutorado em Geotecnia) - Universidade de São Paulo, São Carlos, 2005.

TRICART, J. Ecodinâmica. 1. ed. Rio de Janeiro: IBGE/SUPREN, 1977. 97p.

TUNDISI, J. G. Recursos hídricos no futuro: problemas e soluções. Estudos Avançados, São Paulo, v. 22, n. 63, p. 7-16, 2008.

ZADEH, L. A. Applications of Fuzzy logic: towards high machine intelligence Quotient systems. 1. ed. Series on environmental and intelligent manufacturing: Prentice Hall, 1997. 448p.

Trabalho enviado em 31/07/2019

Trabalho aceito em 17/04/2020 